\title{
Signalverstärkersystem basierend auf der Pheromon-gekoppelten Bildung eines Fluoreszenzproteins in Hefen
}

\author{
Alfred Kick' , Julia Lenhart', Lisa Heike Tranelis², Julia Döring ${ }^{2}$, Maria Patschin², Kai Ostermann², \\ Michael Mertig ${ }^{1,3}$ \\ ${ }^{1}$ Kurt-Schwabe-Institut für Mess- und Sensortechnik Meinsberg e.V., Waldheim, Deutschland \\ ${ }^{2}$ Umweltmonitoring und Endokrinologie - Fakultät Biologie, Technische Universität Dresden, Deutschland \\ ${ }^{3}$ Physikalische Chemie, Technische Universität Dresden, Deutschland \\ Kontakt: alfred.kick@ksi-meinsberg.de
}

\section{Einleitung}

Arzneimittelrückstände und deren Metabolite im Wasserkreislaufsystem sind ein weltweites Problem, da diese Stoffe potenziell gesundheitsschädlich sein können [1]. Auf die erste EU-Beobachtungsliste wurde u.a. der Wirkstoff Diclofenac gesetzt [2], eines der weltweit am häufigsten eingesetzten Medikamente, welches zur Familie der nichtsteroidalen Entzündungshemmer/Antirheumatika gehört. Bei oraler Gabe von Diclofenac werden 60 bis $70 \%$ des Wirkstoffs über den Urin ausgeschieden. Die mittlere Diclofenac-Konzentration im Abwasser beträgt 0,11 bis 2,3 $\mu \mathrm{g} / \mathrm{l}$, in Krankenhausabwässern kann sie auf bis zu $6,88 \mu \mathrm{g} / \mathrm{l}$ und in Abwässern der pharmazeutischen Industrie auf $203 \mu \mathrm{g} / \mathrm{l}(0,69 \mu \mathrm{M})$ ansteigen [3].

Ziel der Arbeit ist die Entwicklung eines Sensorprinzips auf Grundlage genetisch modifizierter Hefen [4]. Diese Hefen (Saccharomyces (S.) cerevisiae) sollen in Ganzzellsensoren zur Detektion von Arzneimittelrückständen in Umweltproben [5, 6] genutzt werden. Im Speziellen soll Diclofenac in geringen Konzentrationen sicher nachgewiesen werden können.

Das hier verwendete Sensorprinzip (Abb. 1) beruht auf der Bildung des Hefe-Pheromons [7] ( $\alpha$-Faktor) durch einen genetisch modifizierten Hefestamm, der bei Anwesenheit von Diclofenac den $\alpha$-Faktor, ein Peptid aus 13 Aminosäuren (Trp-His-Trp-Leu-GIn-Leu-Lys-Pro-Gly-GInPro-Met-Tyr), bildet. Hierbei wird der PDR5-Promoter der Hefe genutzt. Der $\alpha$-Faktor führt dann in einem zweiten Stamm zur Aktivierung des FIG1-Promoters und damit zur Bildung eines fluoreszierenden Proteins (mNeonGreen). Die Bildung des $\alpha$-Faktors, bei sonst gleichen Bedingungen, ist im Wesentlichen von der Diclofenac-Konzentration abhängig, die innerhalb der Detektionszeit als konstant angesehen wird. Damit wächst die $\alpha$-Faktor-Konzentration näherungsweise linear mit der Zeit an. Unter diesen Voraussetzungen kann die Bildungsrate des mNeonGreen als proportional zur $\alpha$-Faktor-Konzentration angenommen werden. Damit erhöht sich die Bildungsrate dieses Fluoreszenzproteins mit der Zeit. Durch die Kumulation des $\alpha-$ Faktors in der Zellsuspension erhöht sich auch die Produktion des mNeonGreen, wodurch - neben dem Ansteuern einer großen Zahl entsprechender Zellen - eine Signalverstärkung im Vergleich zur direkten Detektion des Diclofenac über den PDR5-Promoter erreicht werden kann [4]. Wie in Abb. 1 (links) angedeutet, ist es weiter auch möglich, das Signal zu erhöhen, in dem die Zelldichte, insbesondere der Aktorhefe, durch geeignete Kultivierungsbedingungen erhöht wird.

\section{Methoden und Materialien}

\section{Genetisch modifizierte Hefen}

Die Transformation der S. cerevisiae-Hefestämme erfolgte analog zu der Arbeit von Schuller et al. [4]. Es wurde ein Stamm generiert (S. cerevisiae BY4742 $\triangle$ MFa1 [MATa his3 $\Delta 1$ leu2 $\Delta 0$ lys2 $\Delta 0$ ura3 $\Delta 0$ YPL187w::kanMX4]), welcher das Plasmid p426 PDR5-MFa1 enthält. Dadurch produziert der Stamm in Anwesenheit von Diclofenac $\alpha$ Faktor. Entsprechend wurde eine Aktorhefe (S. cerevisiae BY4741 $\Delta$ bar1 $\Delta$ far1 [MATa his3 $\Delta 1$ leu2 $\Delta 0$ met15 $\Delta 0 \quad u$ ra3 $\Delta 0$ YIL015w::kanMX4 YJL157c::natMX6]) mit dem Plasmid p426 FIG1-mNeonGreen entwickelt. Dieser Stamm reagiert mit der Produktion des Fluoreszenzproteins mNeonGreen in Abhängigkeit der Anwesenheit des Pheromons a-Faktor. Somit erfolgt eine indirekte Detektion von Diclofenac. Die Auswertung erfolgt mittels Fluoreszenzspektroskopie.

\section{Kultivierung der Hefestämme}

Die Experimente wurden in Minimalmedium (1,9 g/l HefeStickstoff-Basis ohne Aminosäuren, $5 \mathrm{~g} / \mathrm{l}$ Ammoniumsulfat, $20 \mathrm{~g} / \mathrm{l}$ Glucose) supplementiert mit entsprechenden Aminosäuren durchgeführt. Die Supplementierung für die Aktorhefe erfolgte mit $60 \mathrm{mg} / \mathrm{l}$ L-Histidin, $80 \mathrm{mg} / \mathrm{l} \mathrm{L-Leucin}$ und $20 \mathrm{mg} / \mathrm{l} \mathrm{L-Methionin;} \mathrm{für} \mathrm{die} \mathrm{Sensorhefe} \mathrm{mit} 60 \mathrm{mg} / \mathrm{L}$ LHistidin, 80 mg/l L-Leucin und 30 mg/l L-Lysin (Carl Roth $\mathrm{GmbH}+$ Co. $\mathrm{KG}$ ).

Vorkulturen wurden in SC-Ura Medium (1,9 g/l Minimalmedium mit 1,926 g/l Kaiser SC Ura Drop-out (ein Medium ohne Uracil; ForMedium ${ }^{\mathrm{TM}}$ ) über Nacht bei $30^{\circ} \mathrm{C}$ im Schüttler bei $180 \mathrm{rpm}$ angelegt. $2 \mathrm{ml}$ der jeweiligen Vorkultur wurde zweimal mit Minimalmedium gewaschen und zentrifugiert (3000 rcf, $7 \mathrm{~min}$ ). Um eine gewünschte Zelldichte (Start-OD 600 ) einzustellen, wurden die optische Absorption bei $600 \mathrm{~nm}$ mit dem NanoDrop-Spektrophotometer (Thermo Fisher Scientific Inc.) in einer Küvette (1 cm Schichtdicke) gemessen und das Pellet im entsprechenden Volumen des Minimalmediums resuspendiert.

\section{Fluoreszenzspektroskopie}

Zur Messung der Fluoreszenzintensitäten der Zellsuspensionen wurden ein Mikroplatten-Reader (Synergy ${ }^{\mathrm{TM}}$ $\mathrm{H} 1$, BioTek Instruments, Inc.) verwendet $\left(30^{\circ} \mathrm{C}\right.$, doppel- 

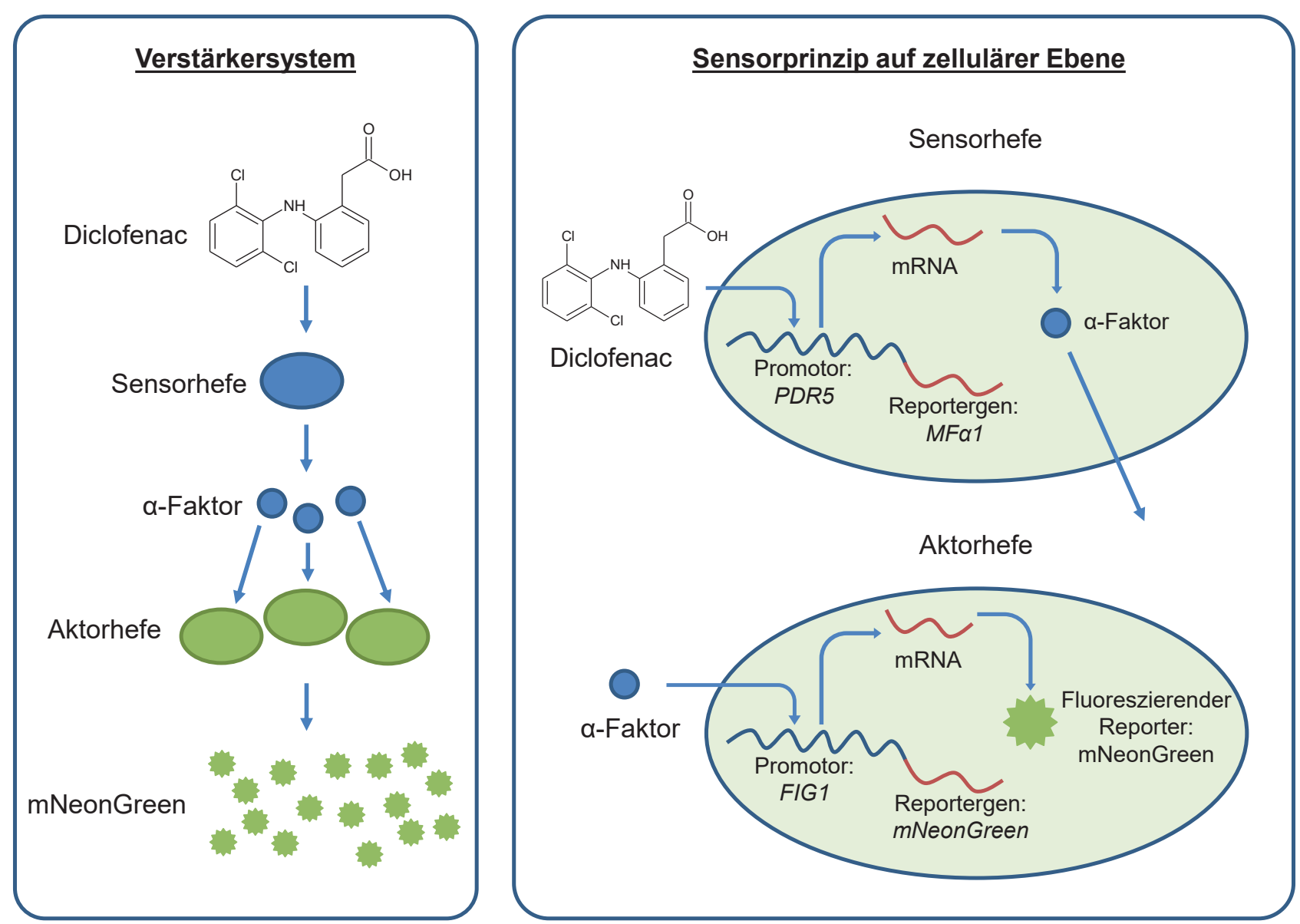

Abb. 1: Prinzip des Signalverstärkersystems und der zelluläre Wirkmechanismus von Diclofenac und $\alpha$-Faktor in den genetisch modifizierten Hefestämmen.

orbitales Schütteln). Je $250 \mu \mathrm{l}$ der Zellsuspensionen wurden in 96-Well-Platten gegeben (F-Boden, BRAND plates $\AA$ - cell Grade ${ }^{T M}$ premium; BRAND GmbH \& Co $K G$ ). Die Wells wurden durch eine gasdurchlässige Membran abgedeckt (Breathe-Easy, Diversified Biotech). Die Fluoreszenz wurde bei $485 \mathrm{~nm}$ Anregungs- und $525 \mathrm{~nm}$ Emissionswellenlänge durch den Boden der Wells ausgelesen. Die Auswertung der Kinetik der Fluoreszenzintensitäten erfolgte als Änderung zum Wert beim Start der Messungen im jeweiligen Well.

\section{Diclofenac im Gemisch von Sensor- und Aktorhefe}

Die Detektion von Diclofenac wurde durch seine Wirkung in einer Mischung von Sensor- und Aktorhefen untersucht. Dabei wurden die Zelldichten von Sensor- und Aktorhefe von OD600 $=0,1$ bzw. OD600 = 1 gewählt. Je 3 Wells wurden mit $250 \mu$ l dieser Hefesuspensionen befüllt wobei Suspensionen mit $1 \mu \mathrm{M}$ Diclofenac (als Natriumsalz; Sigma-Aldrich) und ohne Diclofenac getestet wurden.

\section{Wirkung des $\alpha$-Faktors auf die Aktorhefe}

Zur Prüfung der Wirkung des a-Faktors auf die Aktorhefe, wurden je 3 Wells mit $250 \mu \mathrm{l}$ Hefesuspensionen $\left(\mathrm{OD}_{600}=2\right)$ befüllt wobei Suspensionen mit $50 \mathrm{nM} \alpha-$ Faktor (Zymo Research Corporation) und ohne a-Faktor untersucht wurden.

\section{Separate Nutzung der Sensor- und Aktorhefe}

Bei diesem Experiment wurde 2 Kolben mit je $25 \mathrm{ml}$ Suspension der Sensorhefe $($ Start-OD $600=1)$ über $5 \mathrm{~h}$ bei $30^{\circ} \mathrm{C}$ im Schüttler kultiviert. In einen Kolben wurden zu Beginn $10 \mu \mathrm{M}$ Diclofenac dazugegeben. Danach wurden die Suspensionen zentrifugiert (3000 rcf, $1 \mathrm{~min}$ ) und je $125 \mu$ Überstand mit $125 \mu$ l Aktorhefe (mit frischem Minimalmedium gewaschene Vorkultur, $\mathrm{OD}_{600}=2$ ) gemischt. Damit wurde die Fluoreszenz der Aktorhefe in je 3 Wells mit und ohne vorherige Einwirkung von Diclofenac auf die Sensorhefe untersucht.

\section{Ergebnisse und Diskussion}

Abb. 2 zeigt die Wirkung des a-Faktors auf den Hefestamm, der das Fluoreszenzprotein bildet. Man kann zeigen, dass nach etwa einer Stunde die Fluoreszenzintensität in der Hefekultur mit $50 \mathrm{nM}$ a-Faktor im Vergleich zur Kontrolle ohne $\alpha$-Faktor deutlich ansteigt. Die Fluoreszenzintensität in den Wells ohne $\alpha$-Faktor steigt ebenfalls an; dieser Anstieg ist aber vergleichsweise gering (nach 10 Stunden < 10000 RFU). Er resultiert sehr wahrscheinlich aus der Synthese des Fluoreszenzproteins durch eine basale Aktivität des FIG1-Promotors auch ohne Wirkung des $\alpha$-Faktors. Bei $25 \mathrm{nM} \alpha$-Faktor konnte in Vorversuchen nur eine schwache Erhöhung der Fluoreszenzintensität nach $6 \mathrm{~h}$ detektiert werden. Dies deutet darauf hin, 
dass es unter den gegebenen Versuchsbedingungen eine Schwellenkonzentration des a-Faktors $\left(c_{\alpha, s}<25 \mathrm{nM}\right)$ gibt, welche für die Steigerung der $m$ NeonGreen-Bildung notwendig ist.

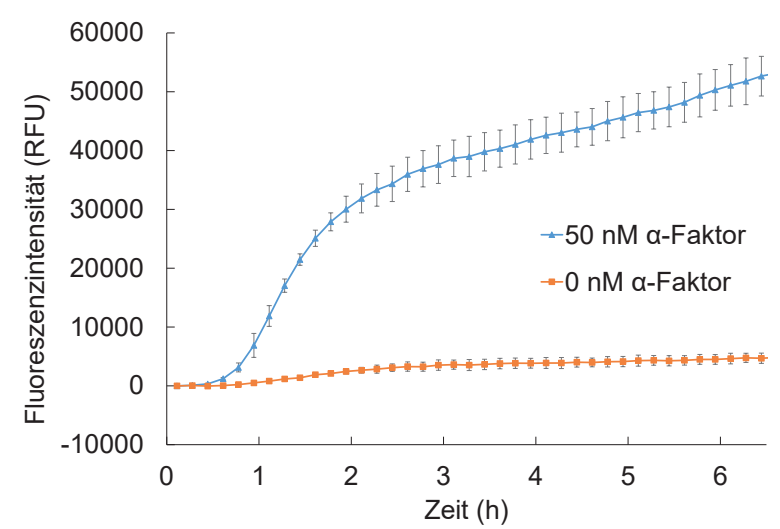

Abb. 2: Kinetik der Fluoreszenzintensität der AktorhefeSuspension bei: $0 \mathrm{nM}$ und $50 \mathrm{nM} \alpha$-Faktor. Start-

$\mathrm{OD}_{600}=2$. Mittelwerte und Standardabweichungen aus je 3 Wells.

Im nächsten Experiment wurde untersucht, ob bei Verwendung einer Mischung aus Sensor- und Aktorhefe die Detektion von Diclofenac möglich ist. Dazu wurde die Menge der Sensorhefe auf $10 \%$ der Menge an Aktorhefe eingestellt, um die Konzentration des basal produzierten a-Faktors in Abwesenheit von Diclofenac gering zu halten. Jedoch erkennt man in Abb. 3, dass in diesem Fall trotzdem eine deutliche Produktion des Fluoreszenzproteins stattfindet. Diese ist zwar deutlich geringer als im Medium mit $1 \mu \mathrm{M}$ Diclofenac, begrenzt jedoch die angestrebte Quantifizierung von kleineren Diclofenac-Konzentrationen in Wasserproben unbekannter Zusammensetzung.

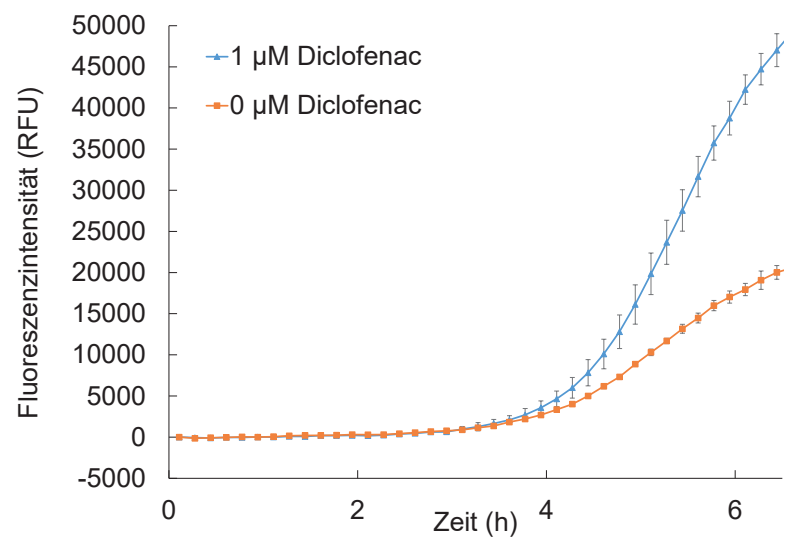

Abb. 3: Kinetik der Fluoreszenzintensität in Suspensionen aus Sensorhefe $\left(\right.$ Start-OD $\left.{ }_{600}=0,1\right)$ und Aktorhefe $($ Start-OD $600=1)$; ohne Diclofenac oder mit $1 \mu \mathrm{M}$ Diclofenac. Mittelwerte und Standardabweichungen aus je 3 Wells.

$\mathrm{Da}$ in anderen Versuchen (hier nicht gezeigt) auch durch weitere Verringerung des Anteils an Sensorhefe das gleiche Problem auftrat, wurde im Weiteren angenommen, dass ggf. durch die unmittelbare Nachbarschaft der Aktorund Sensorhefezellen in der Suspension lokal eine zu hohe Konzentration an basal produziertem a-Faktor auftritt und dieses eine starke Fluoreszenzprotein-Bildung hervorruft, obwohl die auf das Gesamtvolumen bezogene mittlere $\alpha$-Faktor-Konzentration $\left(\bar{c}_{\alpha}\right)$ kleiner als die bereits erwähnte Schwellenkonzentration ist $\left(\bar{c}_{\alpha}<c_{\alpha, s}\right)$ (Abb. 4).

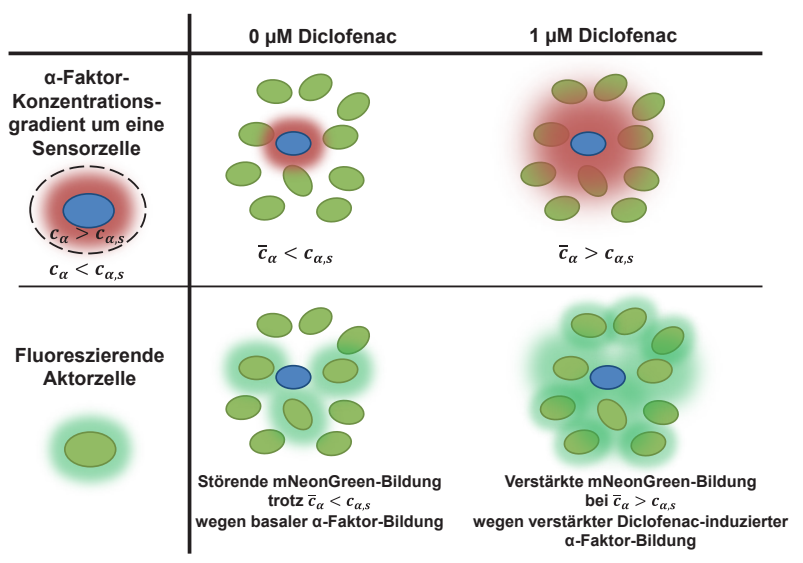

Abb. 4: Veranschaulichung der Konzentrationsverteilung des $\alpha$-Faktors in einer Mischung aus Sensor- und Aktorhefe. $c_{\alpha}-\alpha$-Faktor-Konzentration, $\bar{c}_{\alpha}$ - mittlere $\alpha$ Faktor-Konzentration, $c_{\alpha, s}$-Schwellenkonzentration des $\alpha$-Faktor.

Daher wurde diese Annahme in einem Experiment überprüft, wo die Sensorhefe in Abwesenheit der Aktorhefe zunächst mit Diclofenac versetzt wird. Nach einer bestimmten Einwirkzeit bildet sich in Abhängigkeit der Diclofenac-Konzentration der a-Faktor, welcher dann homogen im Überstand der zentrifugierten Zellsuspension vorliegt. Die Zugabe dieses Überstandes in eine Suspension mit Aktorhefe sollte dann zur Bildung des Fluoreszenzproteins in Abhängigkeit von der $\alpha$-Faktor-Konzentration ohne lokal überhöhte Pheromon-Konzentrationen führen. Die Messwerte in Abb. 5 bestätigen diese Annahme.

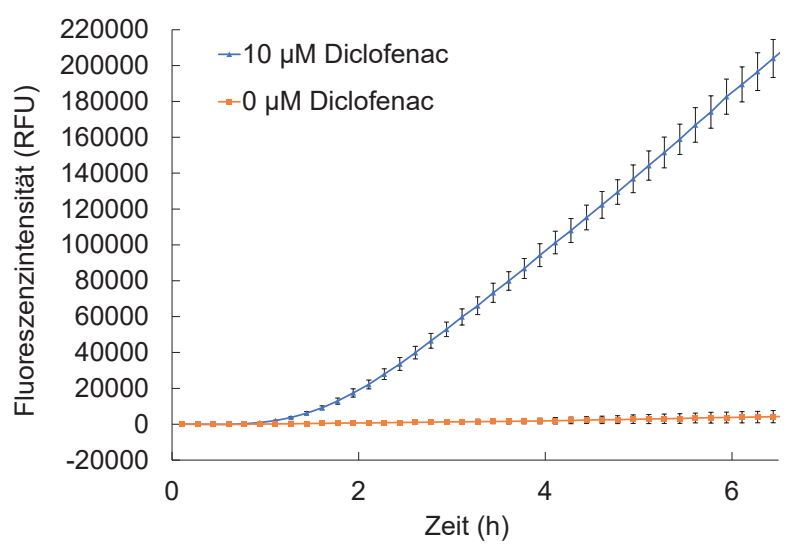

Abb. 5: Kinetik der Fluoreszenzintensität der AktorhefeSuspension nach Zugabe des Überstandes aus einer Sensorhefe-Suspension ( $5 \mathrm{~h}$ Eiwirkzeit mit $0 \mu \mathrm{M}$ bzw. $10 \mu \mathrm{M}$

Diclofenac). Start-OD ${ }_{600}=1$ Mittelwerte und Standardabweichungen aus je 3 Wells. 
Die Konzentration des basal produzierten a-Faktors nach $5 \mathrm{~h}$ ist bei anschließender homogener Verteilung in eine Suspension der Aktorhefe nicht ausreichend, um eine signifikante Erhöhung der Fluoreszenzintensität innerhalb der Detektionszeit zu bewirken. Die entstandene Konzentration an $\alpha$-Faktor beim Einwirken von $10 \mu \mathrm{M}$ auf die Sensorhefe zeigt hingegen bereits nach 1,5 Stunden einen signifikanten Anstieg der Fluoreszenzintensität. Mit diesem Vorgehen kann eine falschpositive Detektion von Diclofenac vermieden werden.

\section{Zusammenfassung}

In dieser Arbeit wird die erfolgreiche Detektion von Diclofenac mittels genetisch modifizierter Hefen durch Implementierung eines $\alpha$-Faktor-gekoppelten Signalverstärkersystems gezeigt. Das Problem der basalen Produktion des a-Faktors wird durch die separate Nutzung von Sensorund Aktorhefen gelöst. Mit der Einwirkzeit des Diclofenacs auf die Sensorhefe von etwa 5 Stunden, kann die Detektion nach spätestens $10 \mathrm{~h}$ ab Zugabe der Diclofenachaltigen Probe abgeschlossen werden.

\section{Literatur}

[1] Umweltbundesamt: Empfehlungen zur Reduzierung von Mikroverunreinigungen in den Gewässern, 2018, https://www.umweltbundesamt.de/sites/default/files/me dien/1410/publikationen/uba_pos_mikroverunreinigung _final_bf.pdf

[2] Richtlinie 2013/39/EU des Europäischen Parlaments und des Rates zur Änderung der Richtlinien 2000/60/EG und 2008/105/EG in Bezug auf prioritäre Stoffe im Bereich der Wasserpolitik, 2013

[3] VIENO, N.; SILLANPÄÄ, M.: Fate of diclofenac in municipal wastewater treatment plant - A review. In: Environ Int 69 (2014), S. 28-39

[4] SCHULLER, A.; RÖDEL, G.; OSTERMANN, K.: Tuning the Sensitivity of the PDR5 Promoter-Based Detection of Diclofenac in Yeast Biosensors. In: Sensors 17 (2017), S. 1506-1522

[5] SCHIRMER, C.; POSSECKARDT, J.; KICK, A.; REBATSCHEK, K.; FICHTNER, W.; OSTERMANN, K.; SCHULLER, A.; RÖDEL, G.; MERTIG, M.: Encapsulating genetically modified Saccharomyces cerevisiae cells in a flow-through device towards the detection of diclofenac in wastewater. In: J Biotechnol 284 (2018), S. $75-83$

[6] SCHIRMER, C.; J. POSSECKARDT, J.; SCHRÖDER, M.; GLÄSER, M.; HOWITZ, S.; SCHARFF, W.; MERTIG, M.: Portable and low-cost biosensor towards onsite detection of diclofenac in wastewater. In: Talanta 203 (2019) 242-247

[7] GROß, A.; RÖDEL, G.; OSTERMANN, K.: Application of the yeast pheromone system for controlled cell-cell communication and signal amplification. In: Lett Appl Microbiol 52 (2011), S. 521-526

\section{Danksagung}

Die vorgestellten Arbeiten sind Teil eines vom Europäischen Fond für Regionale Entwicklung (EFRE) gefördert Projektes (FKZ 100388387). Die Autoren bedanken sich bei der SAB für die finanzielle Unterstützung. Das KurtSchwabe-Institut für Mess- und Sensortechnik Meinsberg e.V. wird mitfinanziert durch Steuermittel auf der Grundlage des vom Sächsischen Landtag beschlossenen Haushaltes. 